\title{
High performance Al bi-layer wire-grid polarizer for deep-ultraviolet to infrared: modeling and design
}

\author{
Li Wang*a ${ }^{\mathrm{a}}$, Harun H. Solak ${ }^{\mathrm{c}}$, and Yasin Ekinci ${ }^{\mathrm{a}, \mathrm{b}}$ \\ ${ }^{a}$ Laboratory for Micro- and Nanotechnology, Paul Scherrer Institute, \\ CH-5232 Villigen PSI, Switzerland; \\ ${ }^{\mathrm{b}}$ Laboratory of Metal Physics and Technology, Dept. of Materials, ETH Zürich, \\ CH-8093 Zürich, Switzerland; \\ ${ }^{\mathrm{c}}$ Eulitha AG, CH-5232 Villigen, Switzerland
}

\begin{abstract}
Metallic wire-grid polarizers (WGP) transmit TM-polarized light (transverse magnetic) and reflect TE polarization (transverse electric) efficiently. They are compact, planar and compatible with integrated circuit (IC) fabrication, which simplifies their use as optical components in nanophotonic, fiber optic, display, and detector devices. In this work, Al bilayer WGPs were designed and numerically simulated using finite element methods. Optical properties of the polarizers were analyzed in the deep-ultraviolet (DUV) to infrared (IR) regions. It was observed that Al bi-layer WGPs show broadband and high TM transmission and extinction ratio. A comparison of the performances of single and bi-layer WGPs show that the latter is highly advantageous over the former one. An extensive study of the dependence of the optical properties of single and bi-layer WGPs on structural parameters, such as period, metal thickness, and, duty cycle (DC), is provided. Optimal structural parameters are obtained within the feasible parameters in terms of nanofabrication. An Al bi-layer polarizer with a period of $80 \mathrm{~nm}$ and a metal layer thickness of $40 \mathrm{~nm}$ showed transmission up to $80 \%$ and extinction of $40 \mathrm{~dB}\left(10^{4}\right)$ and broadband polarizing behavior down to a wavelength of $250 \mathrm{~nm}$.
\end{abstract}

Keywords: Polarization, Metallic nanostructures, Nanowire, Grating, Wire-grid, Transmission, Reflection

\section{INTRODUCTION}

Polarizers are essential optical elements in most optical systems and networks. ${ }^{1}$ Especially, polarizers for UV and visible spectral regions have broad applications in, for instance, liquid crystal displays (LCDs) and various metrology instrument. ${ }^{2,3}$ For visible light, polymer-based polarizer, such as Polaroid (low performance, compact, low cost, broad acceptance angle), ${ }^{4}$ is used, but their operation range is limited to visible and NIR ranges and they are not suitable for demanding applications where high extinction ratio or high power stability are needed. Another alternative is crystalbased polarizers, such as Glan-Thompson type, for visible and UV light above $200 \mathrm{~nm}$. However, this type of polarizer is rather expensive and bulky and has low acceptance angle, and they are used for high-performance applications, such as lab instruments or sensitive and high-quality optical devices. ${ }^{4}$

Metallic wire-grids polarizer, see Fig. 1(a), which transmit TM-polarized light and reflect TE polarization, offers an alternative to these two types. ${ }^{5}$ They are compact and planar and can have high performance (i.e. high transmission and polarization factors). They are also compatible with integrated circuit fabrication, which is advantageous for their use in nanophotonics, displays, and detector devices. They are widely used in radio, microwave, and IR regions of the electromagnetic spectrum. ${ }^{6,7}$ The lower limit for the operation wavelength is defined by the period of the structure and is typically three times the wire grid periods.

Further author information: Send correspondence to Li Wang (li.wang@psi.ch)

Nanophotonics IV, edited by David L. Andrews, Jean-Michel Nunzi, Andreas Ostendorf, Proc. of SPIE Vol. 8424, 842429 - (c) 2012 SPIE · CCC code: 0277-786X/12/\$18

doi: $10.1117 / 12.921837$

Proc. of SPIE Vol. $8424842429-1$ 
With the advance of nanotechnology, WGPs for the visible wavelength region have become technically feasible and affordable for various applications with high performance requirements. The WGPs have been extensively studied, and most of them are fabricated by laser interference lithography or nanoimprint lithography (NIL) and need both metal reactive ion etching and lift-off.,

In our recent work, we demonstrated a new kind of WGP (see Fig. 1b), namely bi-layer metal wire-grids, ${ }^{10}$ which could be obtained by evaporating a metal film onto a dielectric grating, leading to two metal gratings separated by a certain distance and laterally shifted by a half period. Since the fabrication process involves only photoresist patterning and metal evaporation, fabrication of bi-layer metal gratings in this way is much simpler and less costly than a single layer grating. ${ }^{11}$ The previously reported bi-layer polarizers fabricated with EUV interference lithography (EUV-IL) with a maximum transmission of $50 \%$ and a polarization factor of $40 \mathrm{~dB}\left(10^{4}\right)$ was realized without optimization for visible range in both experiment and simulation, which still provide higher optical performance than bi-layer WGPs fabricated by roll-to-roll nano-imprint lithography in Ref $11 .{ }^{10}$ However, the performance of the polarizers, such as TM transmission, specifically in the UV spectral region is fairly low, which can be thus increased by optimization of the structural parameters, such as periods, duty cycles, spacing layer thicknesses, and metal thicknesses, within the range of parameters feasible with currently available nanofabrication techniques.

In this paper, we present the modeling and design of WGPs for the DUV-visible-IR spectral regions. We also compared the performance of bi-layer with single-layer WGPs by simulating a wide variety of geometrical parameters. It is found that bi-layer WGP show similar TM transmission but one or two orders of magnitudes higher extinction ratio than single-layer WGPs. Based on the analysis and feasible nanofabrication parameters, a broadband bi-layer WGP was proposed and the simulation results indicated that an average extinction ratio over $40 \mathrm{~dB}$ and transmission efficiency over $70 \%$ could be achieved down to $250 \mathrm{~nm}$ wavelength.

\section{SIMULATION MODELS}

The electromagnetic properties of the metallic wires were simulated in DUV to IR region with a finite element method in the frequency domain using a commercial code (COMSOL). The geometry of the simulation domain consisted of one unit cell of the periodic grating structures for single layer and bi-layer polarizer were shown in Figs. 1(c) and 1(d), respectively. Al was chosen as the material due to its high conductivity, process compatibility, oxidization resistance and wide operating wavelengths. The metallic wires lie parallel to the $z$ axis on the substrate (high-purity fused silica), which has a refractive index of 1.46 and is frequency independent. For bi-layer polarizers, the top metallic wires were patterned on top of an inorganic photoresist, hydrogen silsesquioxane (HSQ), which has better durability than organic polymer phtoresists in DUV spectral range. It is assumed that all the metal wire-grids are infinitely long along the $z$ direction so that a two dimensional model in the $x y$ plane can be used to simplify the simulation. Floquet periodic boundary conditions were employed for the $x$ axes to describe the periodicity. This condition states that the solution on one side of the unit cell equals the solution on the other side multiplied by a complex-valued phase factor. The phase shift between the boundaries was evaluated from the perpendicular component of the wave vector. Due to the continuity of the field, the phase factor would be the same for the transmitted and reflected beams as for the incident waves. Port conditions were used both for specifying the incident wave and for letting the resulting solution leave the simulation domain without non-physical reflections. A monochromatic plane-wave (TE or TM) was let to propagate in the simulation domain along the $-y$ direction through input to the port condition on top of the grating. This port condition also describes the specular reflection of the incident wave from the grating. The transmitted wave propagated further into the substrate along the $-\mathrm{y}$ direction, and was recorded by the port condition at the bottom of the substrate. In a separate simulation, either a single wavelength or a sweep through DUV, visible, and IR region could be employed. The corresponding optical constant of the materials were used for each wavelength. For Al, the optical constants values based on reflectance data on clean and unoxidized films prepared and measured in UHV were used. ${ }^{12}$ Also, for simplicity, no natural $\mathrm{Al}$ oxide layer was taken into account. 

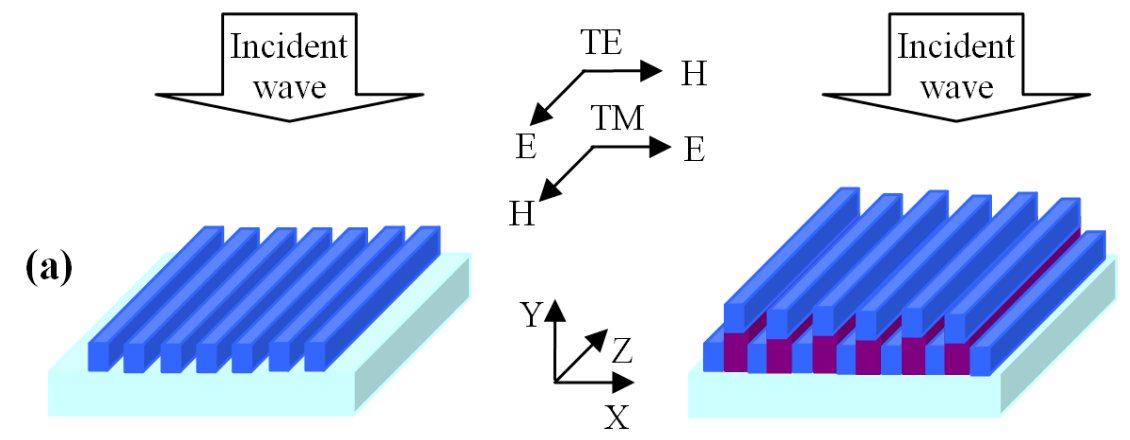

(b)

(c)

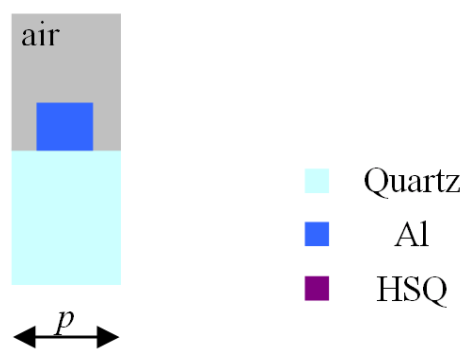

(d)
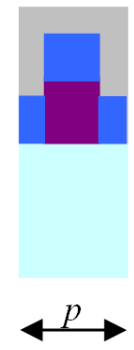

Figure 1. Schematic illustration of a single layer (a) and bi-layer (b) Al wire-grid polarizer. The geometry of the simulation cell is shown in (c, d) for single and bi-layer polarizer, respectively. In (c), the unit cell has one period $p$, Al thickness $t$, width $w$, and DC of $f=w / p$. In (d), the unit cell has one period $p$, photoresist height $h$ and width $w$, top Al layer thickness $t$ with DC of $f=w / p$, and bottom Al layer thickness also $t$ and width (1-f)p/2. The separation between the two Al layers is defined by the thickness of the Al and HSQ layers, $d=h-t$.

\section{SIMULATION RESULTS AND ANALYSES}

\subsection{SINGLE AND BI-LAYER WPGs WITH DIFFERENT PERIODS}

In this part we present the simulation results for both single and bi-layer WGPs with different periods. As a rule of thumb, the shortest operating wavelength for a WGP to work efficiently as a polarizer is about three times the period. The long wavelength operation in principle is not limited and in practice it is limited by the absorption of the substrate. Current commercially available WGP has a period of about $150 \mathrm{~nm}$ due to nanofabrication challenges, which limits it to an effective operating wavelength down to around $450 \mathrm{~nm}$, and an extinction ratio of about 500 to 2000 in the visible wavelength band. ${ }^{1,9,13}$ In this work, we aim for polarizers operating at shorter wavelengths. To realize WPGs enabling effective polarization down to the DUV wavelength ranges, polarizers with period from 60 to $120 \mathrm{~nm}$ were considered and calculated, in which the wire thickness, and DC were kept constant at $40 \mathrm{~nm}$ and 0.5 , respectively. In particular, for bi-layer polarizers, the photoresist (HSQ) height was kept at $60 \mathrm{~nm}$ according to feasible nanofabrication techniques.

The optical transmittances of TM and TE modes as well as the extinction ratios of both single layer and bi-layer polarizers with various periods are shown in Fig. 2. It is worth to note that in Fig. 2 single layer polarizers have only $10 \%$ higher TM transmission than bi-layer ones, but their extinction ratios are 100 times less. Although in Fig. 1, bilayer WGPs have practically two polarizers in-tandem in a first approximation, and the extinction ratio is better than that of a single layer WGP due to near-field coupling. The transmission efficiency, however, is generally reduced because of the same reasons. Nevertheless, a bi-layer WGP grating is not effectively two WGP gratings in-tandem because of the strong near-field coupling and Fabry-Perot effects between the two separate Al wire layers. ${ }^{10}$ This addresses another advantageous of the bi-layer polarizer despite of the easier fabrication and less costly over single layer ones. In Fig. 2, one could also find out that the smaller the period is, the polarizer has higher TM transmission and lower TE 
transmission. Moreover, at the shorter wavelengths, e.g. below $150 \mathrm{~nm}$, strong modulation of TM and TE transmission and thereby extinction ratio occurs owing to the diffraction effects when the incoming wavelength reaches the order of the periods of the polarizers. Therefore, for larger periods, such modulations are expected at longer wavelengths and reduce the polarizing performance. On the other hand, smaller periods are technically difficult in terms of nanofabrication, especially over large-area for commercial applications. Thus, considering feasible nanofabrication capability, and the performance of polarizers in Fig. 2, an $80 \mathrm{~nm}$ period is suitable for our high performance WGPs for DUV to IR. This period is selected in further simulation, which is presented in the following sections.

(a)
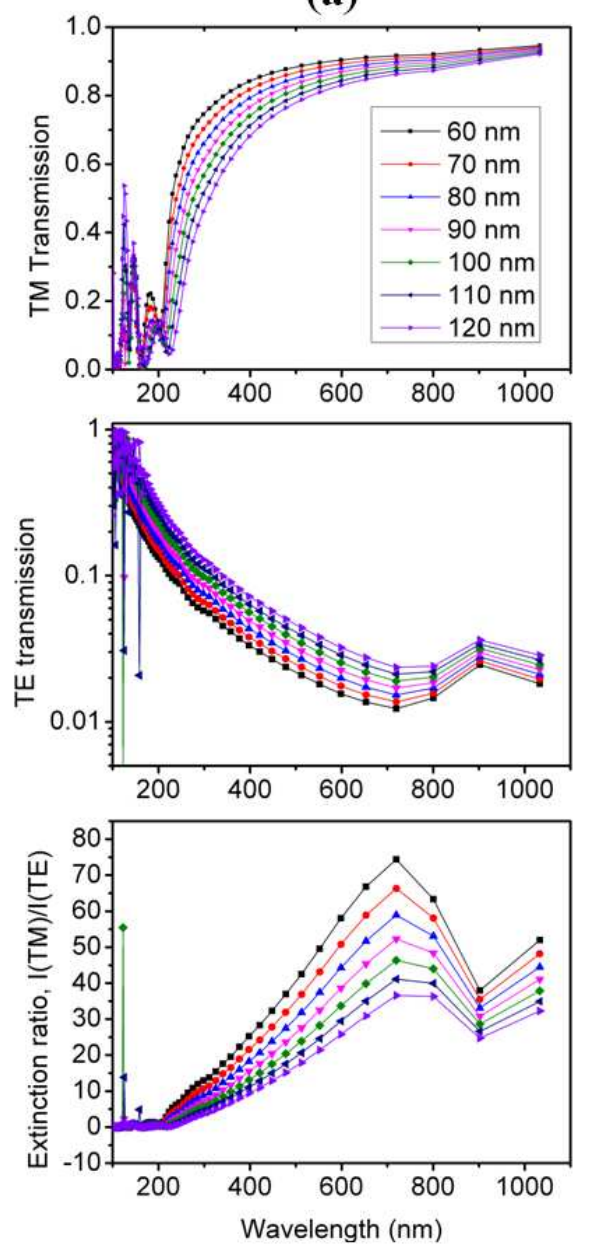

(b)
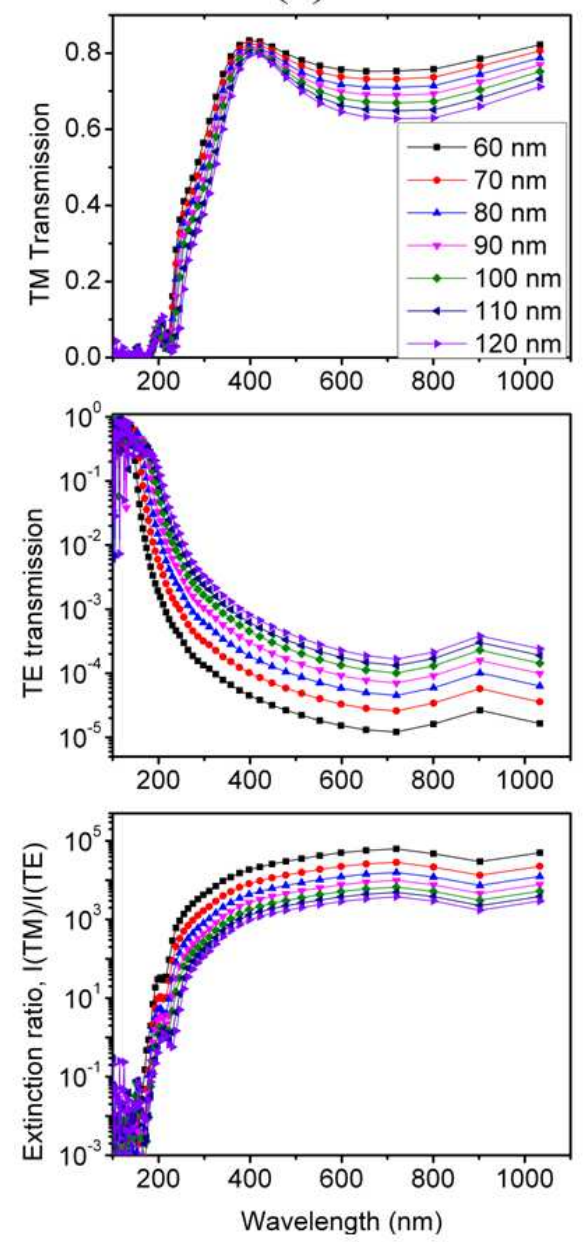

Figure 2. Simulation results of the TM and TE transmittances as well as the extinction ratios of single layer (a) and bi-layer (b) WGPs with various periods.

\subsection{SINGLE AND BI-LAYER WPGs WITH DIFFERENT DCs}

Single layer and bi-layer Al WGPs with different DC (from 0.2 to 0.8 ) were calculated, and the transmittances of TM and TE modes as well as extinction ratios are shown in Fig. 3. The Al wire thickness, and periods were kept at 40 and 80 $\mathrm{nm}$. For single layer Al WGPs, with increasing the DC, the TM transmission efficiencies and the modulation at shorter wavelength decreased, while the extinction ratios increased. For bi-layer WGPs, as the two Al layer complement to each other, their TM transmission efficiencies and extinction ratios first increase and then decrease with the DC, and reach the 
maximum values at DC of 0.6. Therefore, to obtain a good transmission efficiency and high extinction ratio, Al WGPs with DCs around 0.5-0.6 is desirable. In general, as can be seen in Fig. 3, bi-layer Al WGPs provide two orders of magnitude higher extinction ratios and comparable TM transmission efficiencies than that of single layer Al WGPs.

(a)
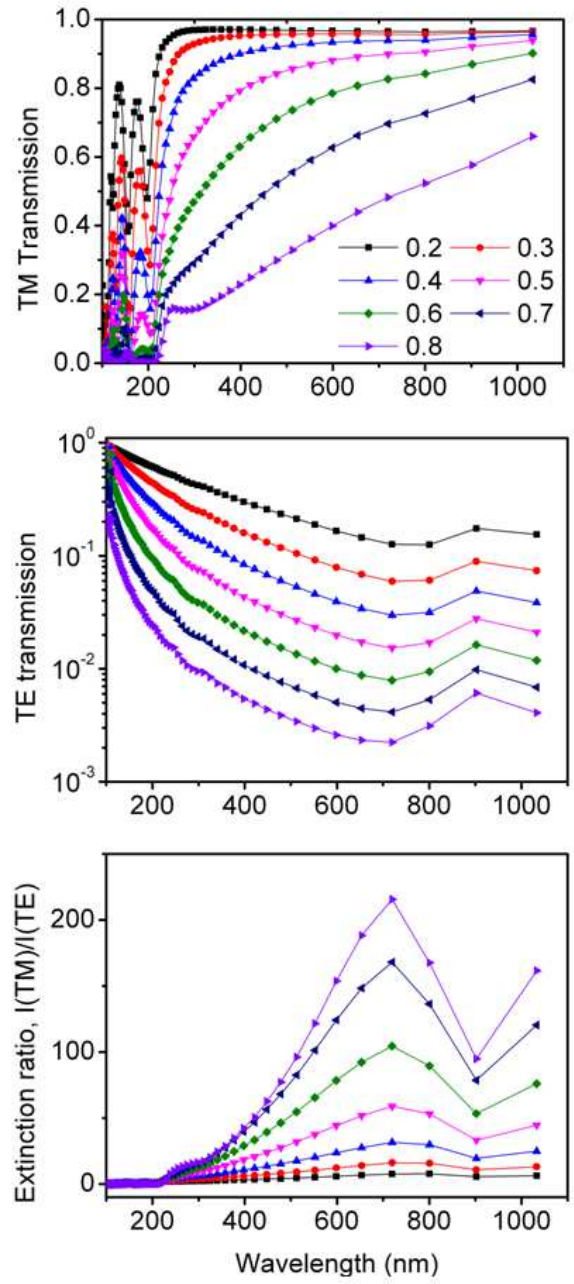

(b)
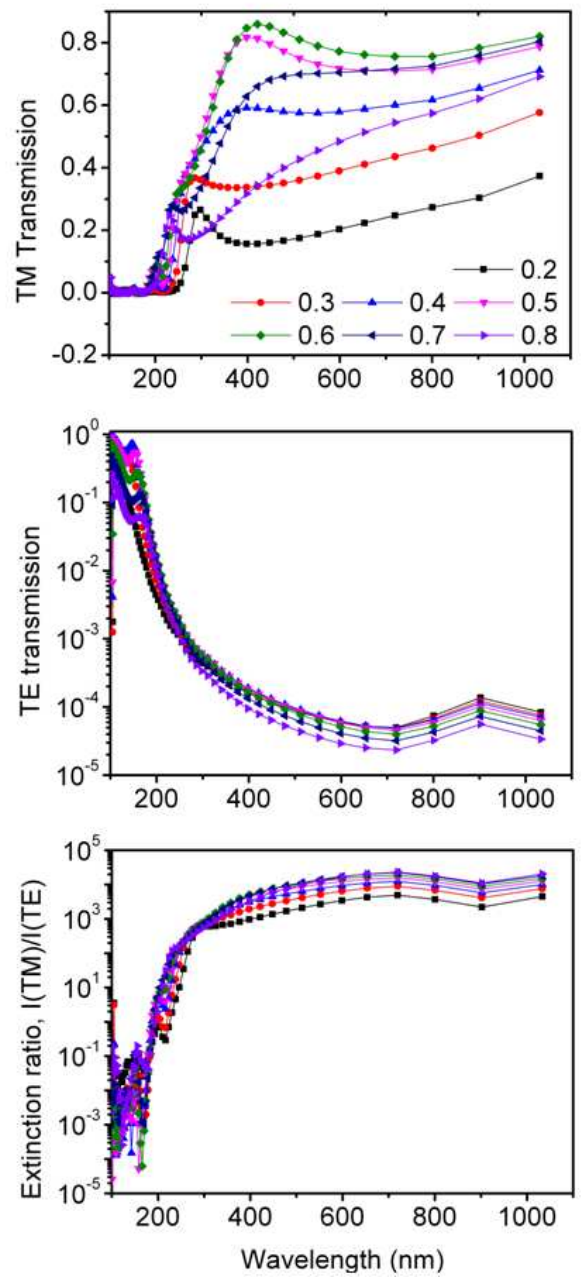

Figure 3. Simulated results of single (a) and bi-layer (b) WGPs with different DCs. The Al wire thicknesses, and periods were kept at 40 and $80 \mathrm{~nm}$, respectively.

\subsection{SINGLE AND BI-LAYER WPGs WITH DIFFERENT AI WIRE THICKNESS}

Another parameter which influences both single and bi-layer WGPs is the thickness of the metallic wire. In this section, simulation results are presented, where the period and DC of the WGPs were kept constant at $80 \mathrm{~nm}$ and 0.5 , respectively, and the dependence of the polarizing performance on the metal thickness is investigated. The thickness of Al wire determines its aspect ratio because the width of Al wire was kept constant, $80 \times 0.5=40 \mathrm{~nm}$. Considering the feasibility with currently available nanolithographic techniques, for single layer polarizers, the Al wires with thicknesses from $20-80 \mathrm{~nm}$ were calculated. While for bi-layer polarizers, the Al wire thicknesses were ranged from $20-50 \mathrm{~nm}$ based on that the thickness of Al wire should not be thicker than that of HSQ layer, which was kept as $60 \mathrm{~nm}$ in our 
simulations. Fig. 4 shows the transmittance of TM and TE modes and extinction ratios of WGPs with various Al wire thicknesses. For single layer polarizers, the TM transmittances show a similar trend in the visible to IR but increase in DUV range, while their extinction ratios increase with increasing thicknesses. For bi-layer WGPs, the TM transmittances decrease in the visible to IR range but slightly increase in DUV range with increasing Al wire thickness. Their extinction ratios increased by an order of magnitude when the $\mathrm{Al}$ wire thickness goes up by only $10 \mathrm{~nm}$.

(a)
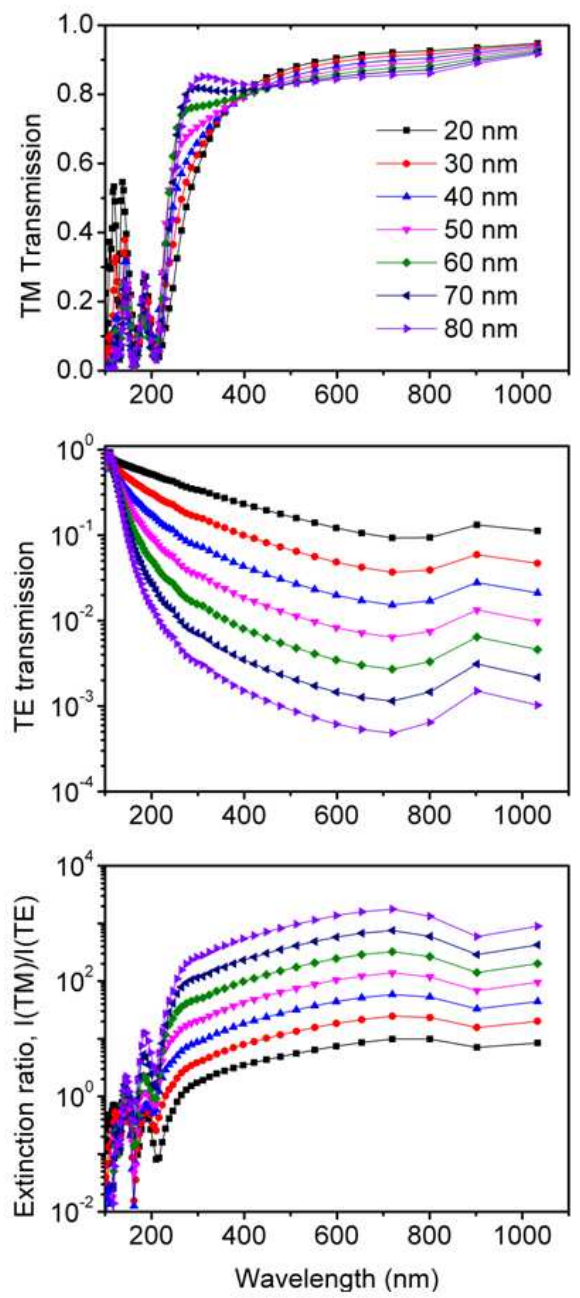

(b)
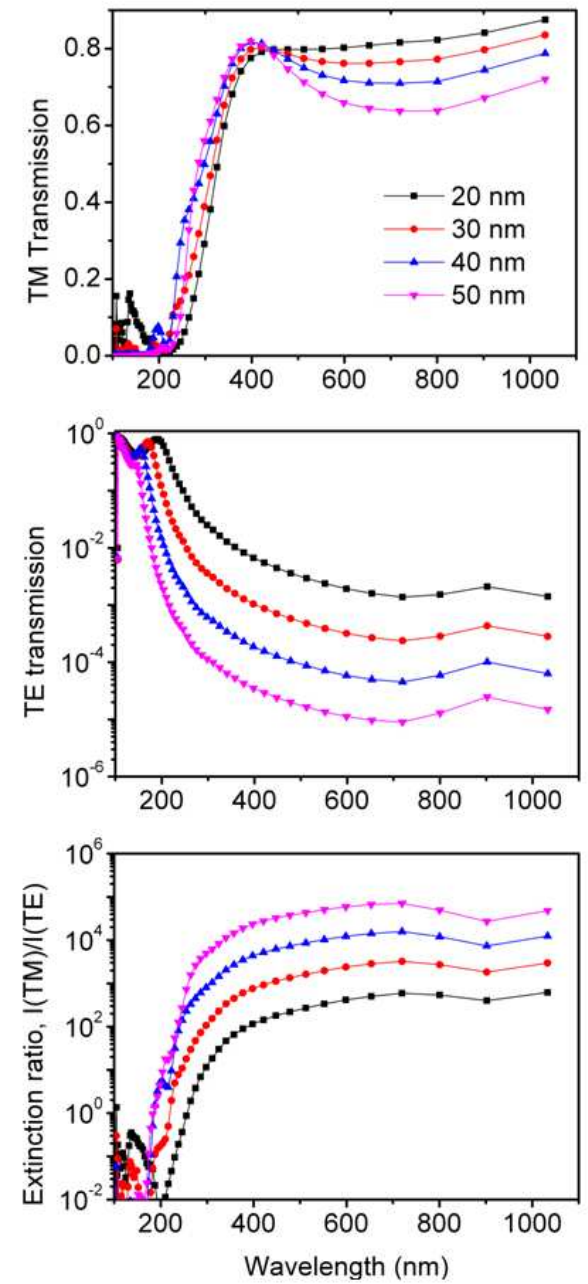

Figure 4. Simulated results of single (a) and bi-layer (b) WGPs with different Al wire thicknesses. In the simulation, the WGP periods and DC were kept at $80 \mathrm{~nm}$ and 0.5 , respectively.

\subsection{BROADBAND AND HIGH-PERFORMANCE BI-LAYER WGP FOR DUV TO IR REGION}

As seen in previous sections, although WGP with a single layer can have high TM transmittance from DUV to IR range, it cannot reach high extinction ratios based on our simulations, except for very thick Al wire, which is technically difficult to achieve. To obtain high optical performance for broadband spectral range, we propose here a bi-layer WGP, as schematically shown in Figs. 1(b), (d) with period of $80 \mathrm{~nm}$, DC of 0.5, and Al wire and HSQ thickness of 40 and 60 
$\mathrm{nm}$, respectively, on high-purity fused silica substrate. The optical transmittances and extinction ratios calculated are shown in Fig. 5 (a). As seen in the figures, this bi-layer polarizer has an average TM transmittance over $70 \%$, and extinction ratio over $40 \mathrm{~dB}$ in the wavelength from IR down to $250 \mathrm{~nm}$. We also show in Fig. 5(b) that this bi-layer WGP has a wide acceptance angle up to 60 and 80 degrees for operation wavelengths of 300 and $700 \mathrm{~nm}$, respectively.

(a)

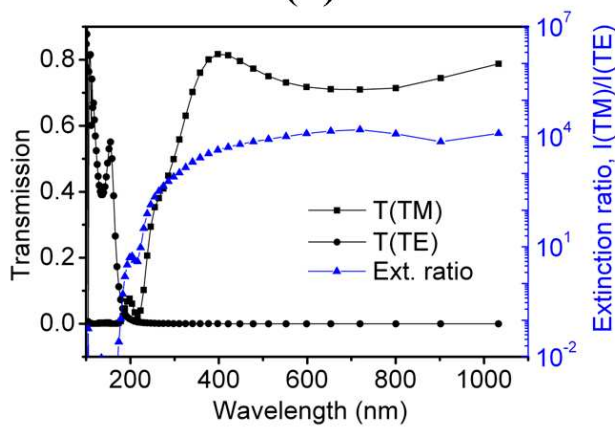

(b)

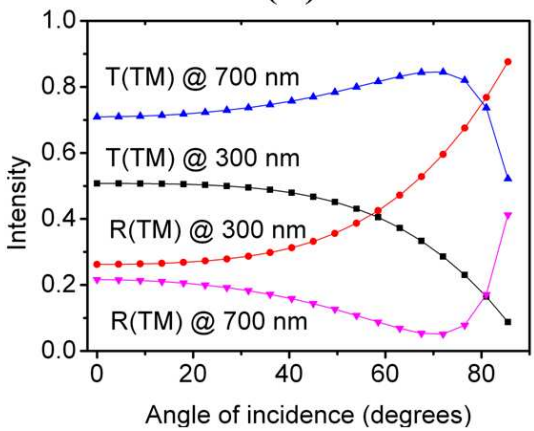

Figure 5. (a) Simulated optical performance results of a proposed bi-layer Al WGP in the DUV to IR spectral regions. (b) Transmission and reflection response of the proposed bi-layer Al WGP for TM incidence at various angles.

\section{CONCLUSIONS AND OUTLOOK}

The optical performance of single and bi-layer WGPs in DUV-IR regions with various structural parameters were studied and compared using finite element methods. In general, small periods enable WGP to work effectively at shorter wavelengths, and provide higher extinction ratio across the operating bandwidth. For both types of polarizers, transmittance is more sensitive to $\mathrm{Al}$ wire DC (linewidth) than to its thickness. However, thicker Al wire grids provide higher extinction ratios. Based on the simulation results, a broadband and high efficiency bi-layer WGP with optimized structural parameters was proposed. The simulation results show that the designed polarizer has an average TM transmittance over $70 \%$, and extinction ratio over $40 \mathrm{~dB}$ in the wavelength from IR down to $250 \mathrm{~nm}$, with a wide incidence acceptance angle. The bi-layer WGP designed in this work is not only cost-effective and simple to fabricate, but also have better performance than single layer WGP or two single layer WGPs in tandem. In addition, since last step of the processes would be metal deposition onto patterned photoresist, the fabrication defects over the large areas, which reduce the polarization factor significantly, could be avoided. The proposed WGP, can be realized by using currently available nanofabrication methods such as nanoimprint lithography, EUV-IL, and laser interference lithography with immersion, Such Al bi-layer wire-grid polarizers can be used as low-cost, high-performance, and broadband polarizers in nanophotonic, fiber optic, display, and detector devices.

\section{ACKNOWLEDGMENTS}

The authors thank Nano-Argovia programme of Swiss Nanoscience Institute Basel (SNI) for financial support.

\section{REFERENCES}

[1] Xu, M., Urbach, H. P., De Boer, D. K. G., and Cornelissen, H. J., "Optical properties of metallic aluminum," Opt. Express 13, 1004 (2005). 
[2] Sonek, G. J., Wagner, D. K., and Ballantyne, J. M., "Optical properties of metallic aluminum," J. Vac. Sci. Technol. 19, 921 (1981).

[3] Tsai, C. C., and Wu, S. T., "Broadband wide-angle polarization converter for LCD backlight," Appl. Opt. 47, 2882 (2008).

[4] Hecht, E., [Optics], 4th ed. Addison-wesley, New York, (2002).

[5] Wang, J. J., Zhang, W., Deng, Z., Deng, J., Liu, F., Sciortino, P., and Chen, L., "High-performance nanowire grid polarizers," Opt. Lett. 30, 195 (2005).

[6] S-W. Ahn, K-D. Lee, J-S. Kim, S. H. Kim, J-D. Park, S-H. Lee, and P-W. Yoon, "Fabrication of a $50 \mathrm{~nm}$ half-pitch wire grid polarizer using nanoimprint lithography," Nanotechnology 16, 1874 (2005).

[7] B. Schnabel, E-B. Kley, F. Wyrowski, "Study on polarizing visible light by subwavelength-period metal stripe gratings," Opt. Eng. 38, 220 (1999).

[8] Wang, J. J., Chen, L., Liu, X. M., Sciortino, P., Liu, F., Walters, F., and Deng, X. G., "30-nm-wide aluminum nanowire grid for ultrahigh contrast and transmittance polarizers made by UV-nanoimprint lithography," Appl. Phys. Lett. 89, 141105 (2006).

[9] Wang, J. J., Walters, F., Liu, X. M., Sciortino, P., and Deng, X. G., "High-performance, large area, deep ultraviolet to infrared polarizers based on $40 \mathrm{~nm}$ line/78 nm space nanowire grids," Appl. Phys. Lett. 90, 061104 (2007).

[10]Ekinci, Y., Solak, H. H., David, C., and Sigg, H., "Bilayer Al wire-grids as broadband and high-performance polarizers," Opt. Express 14, 2323 (2006).

[11] Ahn, S. H., Kim, J. S., and Guo, L. J., "Bilayer metal wire-grid polarizer fabricated by roll-to-roll nanoimprint lithography on flexible plastic substrate," J. Vac. Sci. Technol. B 25(6), 2388 (2007).

[12] Smith, D. Y., [Optical properties of metallic aluminum], in Handbook of Optical Constants of Solids, E. D. Palik, ed. Academic, Orlando, Fla., (1985).

[13] Arnold, S., Gardner, E., Hansen, D., and Perkins, R., "An improved polarizing beamsplitter LCOS projection display on wire-grid polarizers,” SID Int. Symp. Digest Tech. Papers 32, 1282 (2001). 